\title{
Unruh quantization in presence of a condensate
}

\author{
V.A. Belinskii* \\ INFN and ICRA, Rome University "La Sapienza",00185 Rome, Italy, \\ and IHES, F-91440 Bures-sur-Yvette, France \\ N.B. Narozhny ${ }^{\dagger}$, A.M. Fedotov ${ }^{\ddagger}$, and V.D. Mur, \\ Moscow Engineering Physics Institute, 115409 Moscow, Russia
}

We have shown that the Unruh quantization scheme can be realized in Minkowski spacetime in the presence of BoseEinstein condensate containing infinite average number of particles in the zero boost mode and located basically inside the light cone. Unlike the case of an empty Minkowski spacetime the condensate provides the boundary conditions necessary for the Fulling quantization of the part of the field restricted only to the Rindler wedge of Minkowski spacetime.

03.70.+k, 04.70.Dy

The Unruh effect $[1,2]$ consists of the statement that any detector uniformly accelerated in the empty Minkowski space (MS) reveals a universal response as if it were emersed into a thermal bath with the temperature $T_{D U}=g / 2 \pi$, where $g$ is the proper acceleration of the detector ${ }^{1}$. The universality of the detector response is based on interpretation of the Minkowski vacuum state in terms of Fulling-Unruh particles states which appear in the Unruh quantization scheme [2] alternative to the standard plane wave quantization (see also Ref. [3] and citations therein). However, we have shown in our earlier paper [4], see also Refs. [5,6], that the Unruh construction [2] was not a valid quantization scheme in the empty MS. This conclusion stems from the fact that the Unruh scheme neglects the contribution of the singular zero boost mode to the total field amplitude.

The purpose of this paper is to present a situation for which the Unruh quantization procedure could be realized. We argue that this is the case for MS which is not empty but filled with a background of special type. We hope that our consideration will clarify the drawbacks of the Unruh approach to the problem in empty MS. For the sake of simplicity we will restrict our consideration to two-dimensional case.

Let us consider a coherent state for a massive neutral

\footnotetext{
${ }^{*}$ E-mail: belinski@icra.it

${ }^{\dagger}$ E-mail: narozhny@theor.mephi.ru

${ }^{\ddagger}$ E-mail: a_fedotov@yahoo.com

${ }^{1}$ We use natural units $c=\hbar=k_{B}=1$
}

scalar field

$$
|c o h\rangle=Z_{c o h}^{-1 / 2} \exp \left\{\int_{-\infty}^{\infty} \frac{d p}{\sqrt{2 \pi \epsilon_{p}}} f(p) a_{p}^{\dagger}\right\}|0\rangle_{M},
$$

where $a_{p}^{\dagger}$ is a conventional creation operator of a particle in the plane wave state with momentum $p$ and energy $\epsilon_{p}=\sqrt{p^{2}+m^{2}}, f(p)$ is a complex weight function, $Z_{\text {coh }}=e^{\bar{N}}$ is the normalization constant and $\bar{N}$ is the expectation value of total number of particles.

The momentum distribution of particles in the state (1) is described by

$$
d \bar{N}_{p}=\left\langle\operatorname{coh}\left|a_{p}^{\dagger} a_{p}\right| \operatorname{coh}\right\rangle d p=\frac{|f(p)|^{2}}{2 \pi \epsilon_{p}} d p
$$

while the expectation values $\bar{N}$ of the total number of particles and of the field energy $\bar{E}$ in the state (1) are given by Eqs. $(3,4)$ respectively

$$
\begin{aligned}
& \bar{N}=\int_{-\infty}^{\infty} d \bar{N}_{p}=\int_{-\infty}^{\infty}|f(p)|^{2} \frac{d p}{2 \pi \epsilon_{p}}, \\
& \bar{E}=\int_{-\infty}^{\infty} \epsilon_{p} d \bar{N}_{p}=\int_{-\infty}^{\infty}|f(p)|^{2} \frac{d p}{2 \pi} .
\end{aligned}
$$

Clearly, the total number of particles and the field total energy in the state (1) are infinite if the function $|f(p)|^{2}$ decreases too slowly at large momenta. In particular, the integrals in Eqs. $(3,4)$ diverge for the case $f(p)=c=$ const.

When dealing with the Unruh problem it is convenient to quantize the field in the basis of eigenfunctions $\Psi_{\kappa}(x), x=(t, z)$, of the Lorentz boost operator rather than in the plane-wave basis $[2,4]$. These functions have the following integral representation, see, e.g., [4]

$$
\Psi_{\kappa}(x)=\frac{1}{2^{3 / 2} \pi} \int_{-\infty}^{\infty} d q e^{i m(z \sinh q-t \cosh q)-i \kappa q}
$$


and boost destruction operators $b_{\kappa}$ can be expressed in terms of plane wave operators $a_{p}$ as

$$
b_{\kappa}=\int_{-\infty}^{\infty} \frac{d p}{\sqrt{2 \pi \epsilon_{p}}}\left(\frac{\epsilon_{p}+p}{m}\right)^{i \kappa} a_{p} .
$$

The two quantization schemes are unitary equivalent [4].

Let us introduce the notation $|c\rangle$ for the coherent state (1) with $f(p)=c=$ const. Using Eq. (6) it can be easily represented in terms of boost operators as

$$
|c\rangle=Z_{c}^{-1 / 2} e^{c b_{0}^{\dagger}}|0\rangle_{M}
$$

where $b_{0}^{\dagger}$ is the creation operator of a particle in the state corresponding to the singular zero boost mode $\Psi_{0}(x)$,

$$
\Psi_{0}(x)=-i \sqrt{2}^{(+)}(x ; m),
$$

with $\Delta^{(+)}(x ; m)$ being the positive-frequency Whightman function, see Ref. [4]. Thus, in the framework of the boost quantization scheme the state (1) with $f(p)=$ const contains an infinite number of particles concentrated in a single quantum state, namely in the state with zero value of the boost quantum number $\kappa$. Hence, in a sense, this state is equivalent to the Bose-Einstein condensate. It is well known that the Whightman function [which determines the zero mode Eq. (8)] vanishes exponentially fast for large spacelike distances away from the light cone. It is clear therefore that particles of the condensate are located basically inside the light cone. Moreover, the energy density in this state is infinite at the light cone. This is closely related to singular properties of the zero boost mode. Indeed, near the light cone $x_{+} x_{-} \rightarrow 0\left(x_{ \pm}=t \pm z\right)$ the zero mode $\Psi_{0}(x)$ behaves [4] as

$$
\Psi_{0}(x) \sim-\frac{1}{2 \sqrt{2} \pi} \ln \left[-m^{2} x_{+} x_{-}+i \varepsilon \operatorname{sgn}(t)\right]
$$

where we assume that $\varepsilon \rightarrow+0$ and $\operatorname{sgn}(t)=0$, if $t=0$. Therefore even in a one-particle state $\left|B_{0}\right\rangle \propto b_{0}^{\dagger}\left|0_{M}\right\rangle$ the expectation value of energy density $T^{00}$ possesses nonintegrable singularities at the cone, $\left\langle B_{0}\left|T^{00}\right| B_{0}\right\rangle \sim$ $\left(1 / 8 \pi^{2}\right)\left(x_{ \pm}\right)^{-2}, \quad x_{ \pm} \rightarrow 0$. The zero value of the normalization constant $Z_{c}^{-1 / 2}$ in Eq. (7) reflects the well-known fact that the state of condensate belongs to representation of canonical commutation relations (CCR) unitary inequivalent to the conventional Fock representation (see, e.g., $[7])$.

It is worth noting that the state $|c\rangle$ essentially differs from the standard condensate commonly used in condensed matter physics. Since the boost generator does not commute with the Hamiltonian, there is no definite value of energy for any state of the field with a fixed value of $\kappa$ including $\kappa=0$. Therefore the state of the field with $\kappa=0$ is not the ground state of the system, and moreover, it is not stationary. Hence the only common feature of the state $|c\rangle$ and the standard condensate is the infinite number of particles concentrated in a single quantum state. It is clear that the boost condensate $|c\rangle$ could not come into existence for a system of free bosons like it happens in standard situation and should be considered as initially prepared.

It follows from the definition (7) that the state $|c\rangle$ satisfies the relation

$$
b_{\kappa}|c\rangle=c \delta(\kappa)|c\rangle
$$

Let us introduce new operators $\widetilde{b}_{\kappa}$ by

$$
b_{\kappa}=\widetilde{b}_{\kappa}+c \delta(\kappa) \text {. }
$$

These operators satisfy the usual CCR:

$$
\left[\widetilde{b}_{\kappa}, \tilde{b}_{\kappa^{\prime}}^{\dagger}\right]=\delta\left(\kappa-\kappa^{\prime}\right), \quad\left[\widetilde{b}_{\kappa}, \widetilde{b}_{\kappa^{\prime}}\right]=\left[\widetilde{b}_{\kappa}^{\dagger}, \widetilde{b}_{\kappa^{\prime}}^{\dagger}\right]=0
$$

The transformation (11) is often called the boson shift [7]. Let us call the quasiparticles associated with the shifted destruction and creation operators $\widetilde{b}_{\kappa}, \widetilde{b}_{\kappa}^{\dagger}$ boostons. Since

$$
\widetilde{b}_{\kappa}|c\rangle=0
$$

the zero mode condensate $|c\rangle$ can be considered a vacuum state with respect to boostons (of course, it is not vacuum with respect to conventional particles). Hence the boostons can be considered as excitations of the pure condensate state. Such situation is ordinary for condensed matter physics.

Now let us show that it is possible to perform Unruh quantization in the presence of the zero mode condensate. We start with decomposition of the field operator in terms of the boost modes

$$
\phi_{M}(x)=\int_{-\infty}^{\infty} d \kappa\left\{b_{\kappa} \Psi_{\kappa}(x)+\text { h.c. }\right\},
$$

If we apply the boson shift (11) to Eq. (14), the free field $\phi_{M}(x)$ acquires the form

$$
\phi_{M}(x)=\widetilde{\phi}_{M}(x)+\varphi_{c}(x),
$$

where $\varphi_{c}(x)=c \Psi_{0}(x)+$ h.c. is the classical part of the field (the condensate), and

$$
\widetilde{\phi}_{M}(x)=\int_{-\infty}^{\infty} d \kappa\left\{\widetilde{b}_{\kappa} \Psi_{\kappa}(x)+\text { h.c. }\right\},
$$


is the quantized booston field. $c$-number function $\varphi_{c}(x)$ in Eq. (15) has the meaning of the condensate average of the field $\phi_{M}(x)$

$$
\varphi_{c}(x)=\left\langle c\left|\phi_{M}(x)\right| c\right\rangle,
$$

and according to terminology of Ref. [7] forms an "extended macroscopical object". So, in the presence of the condensate the field $\phi_{M}(x)$ describes a system of noninteracting boostons and an extended macroscopical object, compare Ref. [7].

The presence of the extended macroscopical object $\varphi_{c}(x)$ in Eq. (15) allows us to omit the contribution of the zero mode (8) in expansion (16). This means that the booston field can be represented in the form

$$
\widetilde{\phi}_{M}(x) \approx \text { P.v. } \int_{-\infty}^{\infty} d \kappa\left\{\widetilde{b}_{\kappa} \Psi_{\kappa}(x)+\text { h.c. }\right\} .
$$

Physically this approximation means neglecting quantum fluctuations of the zero mode field against the background of the infinite number of particles in the condensate. This can be illustrated symbolically by the following argument. It follows from the commutation relations (12) that $\left[\widetilde{b}_{0}, \widetilde{b}_{0}^{\dagger}\right]=\left.\delta(\kappa)\right|_{\kappa=0}$, so that if $c \neq 0$ then $\left.\widetilde{b}_{0} \sim \sqrt{\left.\delta(\kappa)\right|_{\kappa=0}} \ll c \cdot \delta(\kappa)\right|_{\kappa=0}$. Formally, this estimate should be understood in a weak sense. This means that it is valid for all matrix elements of the operator $\widetilde{b}_{0}$ between the states with finite number of boostons. The matrix elements are supposed to be smeared with respect to the spectral parameter $\kappa$.

Following Unruh [2], we can now split the interval of integration over $\kappa$ in the RHS of Eq.(17) into two for $\kappa=$ $\mu>0$ and $\kappa=-\mu<0$ and after simple transformation arrive to the following form for the field decomposition

$$
\phi_{M}(x)=\int_{0}^{\infty} d \mu\left\{\widetilde{r}_{\mu} R_{\mu}(x)+\widetilde{l}_{\mu} L_{\mu}^{*}(x)+\text { h.c. }\right\}+\varphi_{c}(x) .
$$

Here $R_{\mu}(x)$ and $L_{\mu}(x)$ are the Unruh modes $[2,4]$ and $\widetilde{r}_{\mu}$ and $\widetilde{l}_{\mu}$ are defined as

$$
\begin{aligned}
\widetilde{r}_{\mu} & =\frac{1}{\sqrt{2 \sinh \pi \mu}}\left\{e^{\pi \mu / 2} \widetilde{b}_{\mu}+e^{-\pi \mu / 2} \widetilde{b}_{-\mu}^{\dagger}\right\}, \\
\widetilde{l}_{\mu} & =\frac{1}{\sqrt{2 \sinh \pi \mu}}\left\{e^{\pi \mu / 2} \widetilde{b}_{-\mu}+e^{-\pi \mu / 2} \widetilde{b}_{\mu}^{\dagger}\right\} .
\end{aligned}
$$

The relations (19) are equivalent to the Bogolubov transformation. They define new operators $\widetilde{r}_{\mu}, \widetilde{l}_{\mu}$ which act in the Fock space $\mathcal{H}\left(\widetilde{r}_{\mu}, \widetilde{l}_{\mu}\right)$ unitary inequivalent to the Fock space $\mathcal{H}\left(\widetilde{b}_{\kappa}\right)$. In $\mathcal{H}\left(\widetilde{r}_{\mu}, \widetilde{l}_{\mu}\right)$ the operators $\widetilde{r}_{\mu}, \widetilde{l}_{\mu}$ and their Hermitian conjugates have the sense of destruction, creation operators for $\widetilde{r}$ and $\widetilde{l}$ Unruh quasiparticles which we will call rightons and leftons respectively. The vacuum state from $\mathcal{H}\left(\widetilde{b}_{\kappa}\right)$ (booston vacuum, or condensate state $|c\rangle)$ can be now represented as superposition of $n$-particle states of rightons and leftons $[7,2,4]$

$$
\begin{array}{r}
|c\rangle=Z^{-\frac{1}{2}} \sum_{n=0}^{\infty} \int_{0}^{\infty} d \mu_{1} \ldots \int_{0}^{\infty} d \mu_{n} \exp \left(-\pi \sum_{i=1}^{n} \mu_{i}\right) \\
\times\left|\widetilde{1}_{\mu_{1}}, \ldots \widetilde{1}_{\mu_{n}}\right\rangle_{L} \otimes\left|\widetilde{1}_{\mu_{1}}, \ldots \widetilde{1}_{\mu_{n}}\right\rangle_{R} .
\end{array}
$$

The constant $Z$ in (20) is of course infinite but again this only reflects unitary inequivalence of two Fock spaces. We should emphasize that Eq. (20) is valid only if we are allowed to neglect the contribution of the zero mode to the booston field $\widetilde{\phi}_{M}(x)$. This can be done in MS only in the presence of the condensate and is absolutely inadmissible in empty MS. In the latter case the set of Unruh modes is incomplete, so that the decomposition (18) for the field in empty MS $\left(\varphi_{c}(x)=0\right)$ simply does not exist and the operators (19) have no sense of destruction operators for any particles in MS, see Ref. [4]. Hence the Eq. (20) loses its sense if we change the state $|c\rangle$ by $\left|0_{M}\right\rangle$.

Though Fock spaces $\mathcal{H}\left(\widetilde{r}_{\mu}, \widetilde{l}_{\mu}\right)$ and $\mathcal{H}\left(\widetilde{b}_{\kappa}\right)$ are unitary inequivalent, action of the operators $\widetilde{r}_{\mu}, \widetilde{l}_{\mu}$ on the state vectors from $\mathcal{H}\left(\widetilde{b}_{\kappa}\right)$ are well defined by Eqs. (19) and we can easily derive the Unruh formula $[2,3]$

$$
\left\langle c\left|\widetilde{r}_{\mu}^{\dagger} \widetilde{r}_{\mu^{\prime}}\right| c\right\rangle=\left(e^{2 \pi \mu}-1\right)^{-1} \delta\left(\mu-\mu^{\prime}\right) .
$$

Now, following Unruh [2] we will try to interpret this formula from the point of view of a Rindler observer.

It is highly important that owing to the absence of the zero mode in decomposition (17) the field $\widetilde{\phi}_{M}(x)$ automatically satisfies the condition

$$
\widetilde{\phi}_{M}(0,0)=0,
$$

which follows from the relation $\Psi_{\kappa}(0,0)=(1 / \sqrt{2}) \delta(\kappa)[4]$ and should be understood in weak sense. Note that in the presence of the condensate the field $\phi_{M}(x)$, see Eq. (15), obeys the same condition at the origin $\phi_{M}(0,0)=\infty$ as the classical field $\varphi_{c}(x)$. We have asserted in Ref. [4] that, due to translation invariance, the relation of the type (22) for quantum field in empty MS would mean that all matrix elements of the operator $\phi_{M}(x)$ between physically realizable states are identically equal to zero. Now this assertion does not work since translation invariance of MS is broken in presence of the nonuniform extended macroscopical object, the condensate.

Let us now consider the field $\widetilde{\phi}_{M}(x)$ Eq. (17) restricted to the Rindler wedge of MS, $x=(\eta, \rho), t=$ $\rho \sinh \eta, z=\rho \cosh \eta, \rho>0,-\infty<\eta<\infty$. This field 
as a function of Rindler coordinates satisfies the KleinFock-Gordon equation and obeys the boundary condition $\widetilde{\phi}_{M}(0, \eta)=0$. Hence, as shown in Ref. [4], it can be quantized in accordance with the Fulling procedure [8] which leads to the concept of Fulling quasiparticles. We call here Fulling quanta quasiparticles rather than particles because the classical background $\varphi_{c}(\rho, \eta)$ is not equal to zero, so that the Fulling quanta are excitation over the condensate in the Rindler wedge. In view of boundary condition (22) it follows from Eqs. (5.16) of Ref. [4] that the destruction operator for the Fulling quasiparticle coincides with the destruction operator $\widetilde{r}_{\mu}$ for righton. Therefore the factor in front of $\delta$-function in Eq. (21) can be interpreted as the average number of Fulling quasiparticles seen by an observer moving in the Rindler wedge. Moreover, since $\mu=\omega / g$ and $\eta=g \tau$ where $\omega$ is the energy of Fulling quantum and $\tau$ is the proper time of a uniformly accelerated observer in MS (world line of which lies entirely in Rindler wedge), we arrive to the following conclusion. Any detector moving with a constant proper acceleration in MS filled with zero-mode condensate $|c\rangle$ responds as if it had been immersed into a thermal bath of Fulling quasiparticles at the Davies - Unruh temperature $T_{D U}=g / 2 \pi$.

This statement dramatically differs from the conventional formulation of the Unruh effect $[2,3]$ since the latter assumes acceleration of the detector in the empty MS. The main physical difference between these two situations is that the quantum dynamical degree of freedom associated with zero boost mode, which is an obstacle for validity of Unruh quantization in vacuum, can be naturally ignored in the background singular at the light cone, i.e. when particles occupying the zero mode quantum state form an extended macroscopical object. It is important that neglecting of zero mode contribution to the field amplitude automatically leads to zero boundary condition for the booston field restricted to the Rindler wedge, thus providing a possibility for Fulling quantization.

Certainly concentration of infinite number of particles in a single quantum state is an idealization. Physically, when talking about the zero mode condensate, we should understand not a particular mode but a narrow (with respect to $\kappa$ ) wave packet with the center at $\kappa=0$. It can be seen from the integral representation (5) that at $t=0$ such a wave packet will be narrow with respect to $z$ also. At $t \neq 0$ the wave packet remains narrow in the sense that it has sharp (but not infinite) maxima at the surface of the light cone, $x_{ \pm}=0$. It is clear that expectation value of energy density in this quantum state is not singular any more, compare with the text after Eq. (9). One should keep in mind of course that the wave packet should remain narrow during the time of observation, i.e. the time $\Delta \tau$ which one substitutes instead of $\delta$-function at $\mu=\mu^{\prime}$ in Eq. (21) according to the rule $\delta(0)=\Delta \tau / 2 \pi$. The infinite number of particles in the wave packet state should be understood as macroscopically large. The Unruh construction in presence of such condensate is an approximation of course, but evidently the accuracy of this approximation looks very similar to the accuracy of the well known thermodynamic limit in the many body problems.

We are very grateful to our colleagues A.V. Berkov, S.R. Kelner, Yu.E. Lozovik, B.N. Narozhny and D.N. Voskresensky for helpful discussions. This work was supported by the Russian Fund for Basic Research and by Ministry of Education of Russian Federation.

[1] P.C.W. Davies, J. Phys. A 8, 609 (1975).

[2] W.G. Unruh, Phys. Rev. D14, 870 (1976).

[3] N.D. Birrell and P.C.W. Davies, Quantum Fields in Curved Space (Cambridge University Press, Cambridge, 1982).

[4] N.B. Narozhny, A.M. Fedotov, B.M. Karnakov, V.D. Mur, and V.A. Belinskii, Phys. Rev. D 65, 025004 (2002).

[5] A.M. Fedotov, V.D. Mur, N.B. Narozhny, V.A. Belinskii and B.M. Karnakov, Phys. Lett., A254, 126 (1999).

[6] N. Narozhny, A. Fedotov, B. Karnakov, V. Mur, and V. Belinskii, Ann. Phys. (Leipzig) 9, 199 (2000).

[7] H. Umezava, H. Matsumoto and M. Tachiki, Thermo Field Dynamics and Condensed States (North-Holland, New York, 1982).

[8] S.A. Fulling, Phys. Rev. D7, 2850 (1973). 